\title{
Bibliothek
}

Karsten Schuldt*

\section{Zur Nutzung des Raumes in Wissenschaftlichen Bibliotheken}

\author{
Eine systematische Auswertung von Studien aus den Jahren 2009 bis 2019
}

https://doi.org/10.1515/iwp-2020-2112

Zusammenfassung: Eine systematische Auswertung der Studien, die in den Jahren 2009 bis 2019 dazu publiziert wurden, wie der Raum Wissenschaftlicher Bibliotheken genutzt wird, zeigt einerseits, dass weiterhin das individuelle Arbeiten für akademische Arbeiten überwiegt und andere vermutete Veränderungen nicht in großem Maße stattgefunden haben. Andererseits zeigt sich, dass Bibliotheken vor allem auf sich schauen, Instrumente nicht nachnutzen und fast keine Vergleiche mit anderen Bibliotheken durchführen.

Deskriptoren: Wissenschaftliche Bibliothek, Raum, Nutzung, Studie, Auswertung, Methode, Literaturbericht

\section{The use of space in academic libraries}

A systematic evaluation of studies from the years 2009 to 2019

Abstract: A systematic review of studies on the usage of the user space of acadmic libraries which were published 2009-2019 revealed on the one hand that the individual, silent work on academic tasks is still the main activity. Other assumed changes did not occur in the predicted scale. On the other the review revealed that libraries tend to look just on their own case, did not reuse existing instruments and perform nearly no comparisons with other libraries.

Descriptors: Scientific library, Room, Use, Study, Evaluation, Method, Review

\section{L'utilisation de l'espace dans les bibliothèques univer- sitaires}

Une évaluation systématique des études des années 2009 à 2019

Résumé: Une évaluation systématique des études publiées en 2009-2019 sur l'utilisation de l'espace des bibliothèques universitaires a montré que le travail individuel continue à prédominer pour le travail universitaire et que les autres changements présumés n'ont pas eu lieu dans une large mesure. D'autre part, elle montre que les bibliothèques se regardent surtout elles-mêmes, n'utilisent pas d'instruments et ne font presque pas de comparaisons avec d'autres bibliothèques.

Descripteurs: Bibliothèque scientifique, Salle, Utilisation, Étude, Évaluation, Méthode, Révue

\section{Motivation und Grundfrage}

Wissenschaftliche Bibliotheken beschäftigen sich regelmäßig damit, ihre Benutzungsräume um- oder neuzubauen. Dabei stellen sie immer wieder die Frage, wie sich die Nutzung dieser Räume verändert hat oder noch verändern wird. Gleichzeitig liegen zahlreiche Studien dazu vor, wie diese Räume tatsächlich genutzt werden. Es wäre sinnvoll, wenn Bibliotheken das Wissen aus diesen Studien bei Um- und Neubauprojekten nutzen würden. Um dies zu ermöglichen, wurde eine systematische Analyse dieser Studien durchgeführt. Im Ergebnis zeigt sich, dass sich viele Annahmen über tatsächliche oder zukünftige Entwicklungen bei der Nutzung der Räume Wissenschaftlicher Bibliotheken nicht bewahrheiten haben.

\section{Methode}

Es wurde eine systematische Literaturrecherche durchgeführt, die unter verschiedenen Namen in unterschiedlichen Disziplinen eine eingeführte Methode darstellt. Bei
*Kontaktperson: Dr. Karsten Schuldt, Fachhochschule Graubünden, Schweizerisches Institut für Informationswissenschaft, Pulvermühlestrasse 57, 7000 Chur, Schweiz, E-Mail: karsten.schuldt@fhgr.ch 
dieser wird ein möglichst umfangreicher Korpus relevanter Studien gebildet und dieser anschließend systematisch ausgewertet. Im Idealfall werden Daten aus den analysierten Studien normalisiert und zusammengeführt.

Im vorliegenden Fall wurde wie folgt vorgegangen: Im ersten Schritt wurden in den beiden fachlich relevanten Datenbanken LISA und LISTA sowohl unter ,usage AND space $^{\text {ee }}$ (Ergebnisse LISA: 4689, LISTA: 219) als auch unter „study space AND library" (LISA: 11.671, LISTA: 280) mit der Einschränkung auf die Publikationsjahre 2009-2019 Recherchen durchgeführt. Anschließend wurden die Ergebnislisten intellektuell nach Studien, die in Wissenschaftlichen Bibliotheken die Nutzung des dortigen Raumes untersuchten, aussortiert. Bei den Ergebnissen der Datenbank LISA wurden jeweils die ersten 500 Ergebnisse, bei LISTA alle Ergebnisse überprüft. Da beide Datenbanken erfahrungsgemäs eine gewisse Zeit zwischen $\mathrm{Pu}$ blikation und Anzeige einer Publikation benötigen, wurde Anfang 2020 noch einmal nach aktuell eingegebenen Publikationen recherchiert. Dies ergab drei weitere Studien, die in den Korpus integriert wurden. Insgesamt wurden in diesem ersten Schritt 120 Studien als potentiell thematisch relevant eruiert.

Anschließend wurde ein Raster zur Bewertung der Texte erstellt. Hierzu wurden zehn Prozent (12) der Studien intensiv auf vorkommende Themen hin codiert und diese Codierungen in Kategorien zusammengeführt. Durch dieses Vorgehen wurde das Raster an den Themen orientiert, die in den jeweiligen Studien als erwähnenswert angesehen werden. Es ergaben sich zum Beispiel Cluster zu den Formalia der Texte, zu den verwendeten Methoden, den untersuchten Themenbereichen und den Ergebnissen der Studien. Dann wurden alle Texte gelesen und mit diesem Raster bewertet. In diesem Schritt zeigte sich, wie zu erwarten, dass nicht alle Beiträge wirklich relevante Aussagen zur Fragestellung lieferten (beispielsweise reine Buchbesprechungen darstellten). Insgesamt wurden am Ende für die hier vorliegende Analyse $79 \mathrm{Stu}$ dien aus den Jahren 2009 bis 2020 ausgewertet.

Dieses Vorgehen ließe sich selbstverständlich ausweiten. Beispielsweise erscheinen bibliothekswissenschaftliche und bibliothekarische Zeitschriften, die nicht in den beiden Datenbanken erfasst werden, aber thematisch ausgewertet werden könnten. Ebenso fehlen alle Monographien und Studienabschlussarbeiten. Gleichwohl ist so ein ausreichend großer Korpus entstanden, um Aussagen über Entwicklungen machen zu können.

\section{Ergebnisse}

Die Auswertung der Studien wurden in einer Datenbank erfasst und analysiert. So wurde zum Beispiel sichtbar, wenn mehrere Studien das gleiche Ergebnis hatten oder wenn eine Methode in mehreren Studien genutzt wurde.

\section{Formalia}

Tabelle 1: Anzahl Publikationen pro Jahr.

\begin{tabular}{lr}
\hline Jahr & Anzahl Publikationen \\
\hline 2009 & 3 \\
\hline 2010 & 2 \\
\hline 2011 & 8 \\
\hline 2012 & 1 \\
\hline 2013 & 6 \\
\hline 2014 & 5 \\
\hline 2015 & 9 \\
\hline 2016 & 12 \\
\hline 2017 & 15 \\
\hline 2018 & 5 \\
\hline 2019 & 12 \\
\hline 2020 & 1 \\
\hline
\end{tabular}

Tabelle 2: Anzahl Publikationen pro Land.

\begin{tabular}{lr}
\hline Land & Anzahl Studien \\
\hline USA & 50 \\
\hline Kanada & 13 \\
\hline UK & 8 \\
\hline Australien & 3 \\
\hline Hong Kong & 3 \\
\hline China & 1 \\
\hline Indien & 1 \\
\hline Japan & 1 \\
\hline Libanon & 1 \\
\hline Niederlande & 1 \\
\hline Nigeria & 1 \\
\hline Norwegen & 1 \\
\hline Türkei & 1 \\
\hline
\end{tabular}

Es zeigt sich (Tab. 1), dass das Thema über die Jahre 2009 bis 2019 offenbar an Bedeutung gewann. Das Interesse scheint anzuhalten. Bei den Publikationen findet sich eine kleine Anzahl von Zeitschriften, in denen relevante Studien publiziert wurden (Evidence Based Library and Informa- 
tion Practice: 17 Publikationen, The Journal of Academic Librarianship: 7 und Journal of Library Administration: 5) und die als Kernzeitschriften für diese Fragestellung bezeichnet werden können. Das Thema wird aber auch in bibliothekarischen / bibliothekswissenschaftlichen Publikationen mit allen möglichen Schwerpunkten aufgegriffen.

Ein problematischer Bias ergibt sich, wenn man die Länder vergleicht, in denen die Studien durchgeführt wurden (Tab. 2). Zum Teil durch die unterschiedlichen Publikationskulturen und die Datenbasis (z.B. die gewählten Datenbanken) erklärbar, zeigt sich, dass fast alle Studien im anglo-amerikanischen Raum und dort in den USA durchgeführt wurden. Ein kultureller Bias zum dortigen Universitätssystem - beispielsweise dem Campus-System mit Wohnen vor Ort - lässt sich vermuten.

Weitere Zahlen (hier nicht dargestellt) legen nahe, dass sich nur relativ kleine Teams zu solchen Studien zusammenfinden (im Durchschnitt 2,4 Personen) und dass die meisten Bibliotheken nur einmal solche Studien publizieren (im Korpus findet sich eine Bibliothek dreimal und fünf Bibliotheken zweimal, die übrigen nur einmal). Es ist also eine oft, aber nicht regelmäßig durchgeführte Arbeit.

\section{Methoden}

Tabelle 3: In den Studien benutzte Methoden.

\begin{tabular}{lr}
\hline Benutzte Methoden & Anzahl \\
\hline Umfrage (Online / schriftlich / keine Angabe) & $38(16$ / 8 / 13) \\
\hline $\begin{array}{lr}\text { Beobachtungen (Sweeps / Strukturiert / } \\
\text { Anderes \& keine Angabe) }\end{array}$ & 27 (17 / 8 / 2) \\
\hline $\begin{array}{lr}\text { Interviews (Strukturiert / Semi-Strukturiert / } \\
\text { narrativ / keine Angabe) }\end{array}$ & 15 (2 / 7 / 1 / 5) \\
\hline Ethnographie (davon Photo Exercises) & 12 \\
\hline Head-Count (systematisches Zählen der & 12 \\
\hline Anwesenden) & 10 \\
\hline Andere Methoden & 8 \\
\hline Feedback von Studierenden / Nutzenden per & \\
\hline „Antwortwänden“ & \\
\hline Fokusgruppen & \\
\hline
\end{tabular}

Die Zusammenstellung der in den Studien verwendeten Methoden (Tab. 3) zeigt, dass Bibliotheken einen etablierten Satz an Methoden verwenden, um etwas über die Nutzung ihres Raumes zu erfahren. Nur eine sehr kleine Anzahl wendet sich ungewohnten Methoden zu. 53 dieser Studien kommen zudem mit nur einer Methode aus.

Einiges ist hier bemerkenswert: In 42 der 79 Studien werden - im Text oder im Anhang - die genutzten Instru- mente (Fragebögen, Beobachtungsbögen etc.) mitgeliefert, so dass diese direkt in weiteren Studien nachgenutzt werden könnten. Dies würde es ermöglichen, die erhobenen Daten leicht miteinander zu vergleichen. Aber nur zwei der 79 Studien tun dies. Offenbar entwerfen Bibliotheken immer wieder die notwendigen Instrumente neu. Der Eindruck, dass Bibliotheken auf sich fokussiert sind, wird dadurch verstärkt, dass in 67 der Studien jeweils nur eine Bibliothek untersucht wird. Ein Vergleich von Bibliotheken, die nicht dem gleichen Bibliothekssystem angehören, kommt nur zweimal vor. Auch wenn Bibliotheken offen ihre Ergebnisse und Vorgehensweisen teilen, scheinen sie doch vor allem auf sich bezogen zu agieren.

Interessant ist bei den genutzten Methoden zudem die Verbreitung von sweeps (17), die strukturierte Beobachtung auf festgelegten Wegen und zu festgelegten Zeiten durch die Bibliothek. Zum Einsatz kommen dabei Beobachtungsbögen, auf denen die Zahl der Nutzenden und ihre Aktivitäten festgehalten werden. Diese Methode wird teilweise in den Studien als so bekannt vorausgesetzt, dass sie nicht weiter erläutert werden müssen. Weiterhin auffällig ist, wie oft unterschiedliche Formen von Foto Exercises (9) durchgeführt werden, bei denen Nutzende die Aufgabe erhalten, bestimmte Orte zu fotografieren und diese Bilder anschließend zu diskutieren. Dass mehrere Bibliotheken Whiteboards oder Ähnliches verwenden, um Fragen an ihre Nutzenden zu stellen und anschließend deren Antworten zu analysieren (10), ist deshalb interessant, weil dies in der bibliothekarischen Literatur kaum diskutiert wird, obwohl es offenbar verbreitet ist.

In einer ganzen Reihe von Studien wird zudem behauptet, andere Bibliotheken würden bislang nur zählen, wie viele Nutzende den Raum nutzen. Erst die eigene Studie würde andere Methoden nutzen. Dies ist offensichtlich falsch, wie in der Tabelle 3 sichtbar ist, verdeutlicht aber, dass Bibliotheken vor allem auf sich schauen, wenn sie solche Studien durchführen.

Auffällig ist in diesem Zusammenhang, dass ein Großteil der Studien (73 von 79) sich als explorativ beschreiben lässt: Es geht nicht um Theoriebildung, Methodenentwicklung oder das Testen von Thesen, sondern immer wieder darum, die Situation vor Ort zu erfassen und zu beschreiben. Nur in zwei Studien werden statistische Modelle generiert, ansonsten werden die erhobenen Daten beschreibend dargestellt oder rein verbal präsentiert. Es scheint keine Bestrebungen zu geben, allgemeine Aussagen oder Theorien zur Nutzung des Bibliotheksraumes zu generieren. 


\section{Themen}

Eine kleine Anzahl von Studien fragt demographische (Alter, Geschlecht) oder formale Kriterien (Studienrichtung, Wohnort auf dem Campus, Bachelor/Master/Ph.D.-Student) ab. Allerdings werden die dann erhobenen Ergebnisse einfach dargestellt und keine Erklärung für die jeweilige Verteilung der Daten versucht. Der Großteil der Studien scheint sich nicht für diese Daten zu interessieren, sondern zählt alle Nutzenden gleich.

Tabelle 4: Untersuchte Bereiche.

\begin{tabular}{lr}
\hline Abgefragte Bereiche / Themen & Anzahl \\
\hline Häufigkeit der Bibliotheksbenutzung & 30 \\
\hline Andere Themen & 22 \\
\hline Präferenzen für Sitzgelegenheiten / Arbeitsorte & 21 \\
\hline Lautstärke / Ruhe & 17 \\
\hline Nutzung bestimmter Technologien & 17 \\
\hline Präferenz für bestimmte Möblierung & 16 \\
\hline Zufriedenheit mit der Bibliothek & 14 \\
\hline Mögliche Verbesserungen & 11 \\
\hline Angebote der Bibliothek & 8 \\
\hline Licht & 7 \\
\hline WiFi & 5 \\
\hline
\end{tabular}

Ein Blick auf die Themen, die in den Studien genau untersucht wurden (Tab. 4), zeigt klare Tendenzen. Hierbei muss bedacht werden, dass eine Reihe der ausgewerteten 79 Studien sich mit spezifischen Räumen (z. B. Familienraum, Co-Working Space) oder Nutzungszeiten (z. B. spät abends) beschäftigte. Dies erklärt die „anderen Themen“ (22), die jeweils einmal vorkamen. Auffällig ist, dass sich vor allem für die Häufigkeit der Bibliotheksbesuche und erst danach für die konkrete Nutzung des Raumes interessiert wird.

Hier nicht dargestellt, aber bedeutsam ist, dass die Frage nach der Nutzung bestimmter Technologien im Zeitverlauf merklich abnimmt. Wurde in den beginnenden 2010er Jahren noch gefragt, welche der von den Bibliotheken angeschafften Technologien zur kollaborativen Arbeit genutzt werden oder wie viel Studierende eigne Rechner nutzen, interessiert dies in den späteren Jahren nicht mehr. In den Ergebnissen der Studien ist klar, dass bei den heutigen Nutzenden die Verwendung eigener Laptops vorausgesetzt wird.

Auffällig in dieser Auflistung ist das Fehlen des Bibliotheksbestandes und des Bibliothekspersonals. Kaum eine Studie thematisiert, ob und wie der Bestand genutzt wird oder wie die Nutzenden mit den Bibliothekspersonal interagieren.

\section{Ergebnisse}

Da Bibliotheken in Studien zur Nutzung des Raumes immer wieder neue Instrumente entwerfen und keine Vergleiche anstreben, ist es auch unmöglich, die Daten und Ergebnisse dieser Studien zu normalisieren. Zum Beispiel ist offenbar unterschiedlich definiert, was unter „Arbeit in Gruppen" gemeint ist. In einigen Studien werden offenbar Nutzende, die zusammensitzen, darunter gezählt. In anderen werden die Tätigkeiten der Nutzenden unterteilt und beispielsweise Nutzende, die zusammensitzen, aber nicht zusammenarbeiten, nicht als Gruppe gezählt. In anderen Studien wird dies gar nicht thematisiert, aber dennoch Daten zu Gruppen genannt. Zudem sind die Daten vieler Studien sehr lokal präsentiert. Beispielsweise wird die durchschnittliche Nutzung auf unterschiedlichen Etagen angegeben oder die Nutzung eines speziellen Raumes präsentiert, aber nicht abstrahiert, was diese Ergebnisse allgemein für andere Bibliotheken bedeuten, beispielsweise was für welche Räume die unterschiedlichen Etagen stehen.

Insoweit kann man die Ergebnisse der einzelnen Studien nur auf einer abstrahierten Ebene zusammenführen, nicht auf der Ebene der konkreten Daten. Aber auch diese Zusammenführung (Tabelle 5, weitere Ergebnisse, die einbis dreimal vorkamen, sind hier nicht dargestellt.) liefert interessante Hinweise. 
Tabelle 5: Zusammengefasste Ergebnisse der Studien.

\begin{tabular}{lr}
\hline Häufige Ergebnisse & Anzahl \\
\hline Hauptaktivität individuelles Studieren / Arbeiten & 33 \\
\hline Nutzung vor allem für akademische Arbeiten & 11 \\
\hline Lautstärke wichtig, wird aber unterschiedliche & 10 \\
wahrgenommen & 9 \\
\hline Laptops normal, eigene Technik normal & 9 \\
\hline Mehr Strom, mehr Outlets gewünscht & 8 \\
\hline Essen / Trinken als wichtige Aktivität & 7 \\
\hline Bachelorstudierende als Hauptnutzerinnen \& Hauptnutzer & 7 \\
\hline Nutzung unterschiedlich, alle Nutzungsformen kommen vor & 6 \\
\hline Nutzung Montag bis Mittwoch, Montag bis Donnerstag stark & 6 \\
\hline Mehr Gruppenarbeit als individuelle Arbeit & 5 \\
\hline Je länger im Studium, umso weniger Bibliotheksnutzung & 5 \\
\hline Atmosphäre der jeweiligen Bibliothek wichtig & 5 \\
\hline Soft-seating kaum genutzt / gewünscht & 5 \\
\hline Gewünscht: Platz für Trinken und Essen & 5 \\
\hline Mehr Männer als Frauen & 4 \\
\hline Lautstärke steigt zu Mittag, Hauptnutzung gegen 13:00 Uhr & 4 \\
\hline Positive Rückmeldungen & 4 \\
\hline Zu wenig Gruppenräume & 4 \\
\hline Normale Nutzungsdauer 1-2 Stunden pro Besuch & 4 \\
\hline Studierende wissen, was für sie wichtig ist & 4 \\
\hline
\end{tabular}

Mit 33 Nennungen ist das individuelle Arbeiten die wichtigste Tätigkeit. Dies gilt für alle Bibliotheken unterschiedlichster Größen und Aufgaben sowie für Bibliotheken nach einem Umbau, der häufig Gruppenarbeit unterstützen bzw. fördern sollte (21 Studien wurden für die Evaluation nach dem Um- oder Neubau genutzt). Teilweise zeigten sich die Autorinnen und Autoren explizit über dieses Ergebnis überrascht. Eine kleine Anzahl von Studien (5) widerspricht dieser Erkenntnis. Aber dabei ist Vorsicht geboten: Eine untersuchte explizit einen als Co-WorkingSpace gebauten Raum und auch in diesem ist die individuelle Arbeit verbreitet. Eine zweite Studie fand in einer Kunst- und Design-Universität statt, bei der Studierende in Projekten lernen und dafür die Bibliothek nutzen. Die anderen drei Studien zählen vor allem, ob Nutzende zusammen oder allein sitzen. Das ist, wie andere Studien im Korpus immer wieder zeigen, nicht ausreichend, um Aussagen über die Arbeit in Gruppen zu treffen, da diese sehr unterschiedlich sein kann und sehr wohl individuelles Arbeiten von Nutzenden beinhalten kann.

Auch die mit elf Nennungen zweithäufigste Nutzung, unterstützt diese Erkenntnis: Die Bibliotheksräume werden vor allem für akademische Tätigkeiten (Lernen, Arbei- ten schreiben, Forschen) verwendet. Auch hier zeigen sich einige Autorinnen und Autoren überrascht, dass Studierende die Bibliothek nicht anders nutzen. Bibliotheken erwarteten offenbar, mehr soziale Aktivitäten in ihren Räumen, wie zum Beispiel ein Treffen von Freundinnen und Freunden. Dahinter steht die Idee, dass heutige Studierende verschiedene Tätigkeiten miteinander verbinden. Das bestätigt sich kaum und wird dadurch unterstrichen, dass die durchschnittlich angegebene Nutzungszeiten der Bibliothek rund ein bis zwei Stunden beträgt - also nicht so lange, dass weitere zeitintensive Tätigkeiten aufgenommen werden könnten. Auch fand sich in den Studien, dass die für offene Kommunikation oder Wohnzimmeratmosphäre eingerichteten soft-seating areas mit Sofas und ähnlichen Möbeln ihren gedachten Zweck weit weniger als erwartet erfüllten. Immer kamen in diesen Räumen auch andere Nutzungsformen vor und Tätigkeiten in Gruppen blieben unter den Erwartungen der Autorinnen und Autoren.

Hervorzuheben ist, dass Ruhe beziehungsweise Lärm immer wieder thematisiert wurde. Dabei war aber die Wahrnehmung, was zu laut oder zu leise ist, individuell unterschiedlich. Dies scheint ein ungelöstes Problem zu sein.

Interessant ist zudem, wie oft die Punkte Essen und Trinken angesprochen wurden. Man kann davon ausgehen, dass dies, trotz lokaler Verbote, in allen Bibliotheken praktiziert wird. Auch dies überraschte einige der $\mathrm{Au}$ torinnen und Autoren.

Aufmerksamkeit sollte erregen, dass einige Studien zeigten, dass die Bibliotheksnutzung offenbar mit der Studiendauer abnimmt.

\section{Relevanz}

Die hier beschriebene systematische Analyse hat einige Relevanz für Bibliotheken und die Bibliotheksforschung.

- Für Neu- und Umbauprojekte in Bibliotheken kann es als einigermaßen gesichertes Wissen gelten, dass auch nach umfangreichen Umbauten - und wohl auch entgegen anderslautender Ergebnisse in den oft während der Planung solcher Umbauten durchgeführten Design- oder Partizipationsprozessen - die Hauptnutzung der Bibliothek das individuelle Arbeiten an akademischen Aufgaben mit einer durchschnittlichen Aufenthaltsdauer von ein bis zwei Stunden sein wird. Andere Nutzungsweisen werden vorkommen und sollten ermöglicht werden, aber auf sie sollte nicht der Hauptfokus gelegt werden.

- Seit langem gibt es Vorhersagen dazu, wie sich das Lernen und die Nutzung Wissenschaftlicher Biblio- 
theken verändern wird. Viele Bibliotheken haben auf diese mit Um- und Neubauten reagiert. Die erwarteten Verhaltensänderungen haben offenbar nicht stattgefunden, was einige Autorinnen und Autoren auch erwähnen. Die Wahrscheinlichkeit ist groß, dass sie auch in Zukunft nicht eintreffen werden. Der zukunftssicherste Bibliotheksraum scheint weiterhin einer zu sein, der ermöglicht, was ältere Bibliotheksräume auch ermöglichen. Was sich verändert hat, ist, dass Nutzende für ihre individuellen Arbeiten ihren eigenen Rechner mitbringen und voraussetzen, für diesen Strom und WLAN vorzufinden.

- Für Bibliotheken, die Studien zur Nutzung des Bibliotheksraumes vornehmen möchten, zeigte sich, dass es schon einen recht etablierten Methodensatz und zahlreiche schon entwickelte Instrumente gibt, deren praktischer Nutzen gezeigt wurde. Es ist nicht notwendig, diese noch einmal zu erstellen. Sinnvoll wäre es, sie nachzunutzen, um die eigenen Ergebnisse mit anderen vergleichen $\mathrm{zu}$ können und zu erfahren, ob die eigene Bibliothek Besonderheiten aufweist. Ebenso sinnvoll wäre es, sich nicht auf einzelne Bibliotheken zu konzentrieren, sondern Vergleiche über mehrere Einrichtungen zu organisieren. Dies würde Wissen über Einzelfälle hinaus produzieren.

- Der Bias zum anglo-amerikanischen Raum führt zu zwei Feststellungen: Erstens wäre es wichtig, dass auch Bibliotheken aus anderen Staaten solche Studien publizieren. So würden mögliche kulturelle Unterschiede sichtbar. Zweitens wäre es lohnend, die hier geschilderte systematische Recherche auch für andere Ländern mit ausreichend vielen bibliothekarischen Publikationsmedien durchzuführen, um einen auswertbaren Korpus zu bilden.

- Interessant wäre weiterhin, solche systematischen Recherchen für andere bibliothekarische Themen durchzuführen. So könnte man feststellen, ob Bibliotheken auch bei anderen Fragestellungen vor allem Studien über die eigene Institution durchführen und immer wieder neue Instrumente schaffen und publizieren, ohne eine Methodenentwicklung oder Theoriebildung $\mathrm{zu}$ betreiben. So bleibt viel Potential ungenutzt, um vergleichbares Wissen zu schaffen, das die Entscheidungsfindung der Bibliotheken vereinfachen könnte.

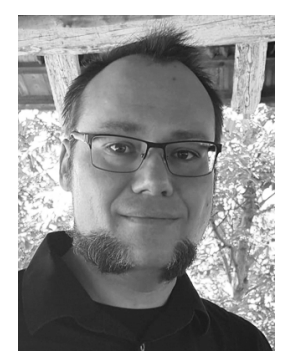

\section{Dr. Karsten Schuldt}

Fachhochschule Graubünden

Schweizerisches Institut für

Informationswissenschaft

Pulvermühlestrasse 57

7000 Chur

Schweiz

karsten.schuldt@fhgr.ch

Dr. Karsten Schuldt, Wissenschaftlicher Mitarbeiter Schweizerisches Institut für Informationswissenschaft, FHGR. Redakteur LIBREAS. Library Ideas. 